\title{
Implementação e avaliação de um sistema de gerenciamento de imagens médicas com suporte à recuperação baseada em conteúdo*
}

\author{
Implementation and evaluation of a medical image management system \\ with content-based retrieval support \\ Edilson Carlos Caritá ${ }^{1}$, Enzo Seraphim ${ }^{2}$, Marcelo Ossamu Honda ${ }^{3}$, Paulo Mazzoncini \\ de Azevedo-Marques ${ }^{4}$
}

Resumo OBJETIVO: Neste artigo são descritas a implementação e avaliação de um sistema de gerenciamento de imagens médicas com suporte à recuperação baseada em conteúdo (PACS-CBIR), integrando módulos voltados para a aquisição, armazenamento e distribuição de imagens, e a recuperação de informação textual por palavras-chave e de imagens por similaridade. MATERIAIS E MÉTODOS: O sistema foi implementado com tecnologias para Internet, utilizando-se programas livres, plataforma Linux e linguagem de programação C++, PHP e Java. Há um módulo de gerenciamento de imagens compatível com o padrão DICOM e outros dois módulos de busca, um baseado em informações textuais e outro na similaridade de atributos de textura de imagens. RESULTADOS: Os resultados obtidos indicaram que as imagens são gerenciadas e armazenadas corretamente e que o tempo de retorno das imagens, sempre menor do que 15 segundos, foi considerado bom pelos usuários. As avaliações da recuperação por similaridade demonstraram que o extrator escoIhido possibilitou a separação das imagens por região anatômica. CONCLUSÃO: Com os resultados obtidos pode-se concluir que é viável a implementação de um PACS-CBIR. O sistema apresentou-se compatível com as funcionalidades do DICOM e integrável ao sistema de informação local. A funcionalidade de recuperação de imagens similares pode ser melhorada com a inclusão de outros descritores.

Unitermos: CBIR; PACS; DICOM; Imagens médicas; Sistemas de informação em saúde.

Abstract OBJECTIVE: The present paper describes the implementation and evaluation of a medical images management system with content-based retrieval support (PACS-CBIR) integrating modules focused on images acquisition, storage and distribution, and text retrieval by keyword and images retrieval by similarity. MATERIALS AND METHODS: Internet-compatible technologies were utilized for the system implementation with freeware, and $\mathrm{C}++$, PHP and Java languages on a Linux platform. There is a DICOM-compatible image management module and two query modules, one of them based on text and the other on similarity of image texture attributes. RESULTS: Results demonstrate an appropriate images management and storage, and that the images retrieval time, always < $15 \mathrm{sec}$, was found to be good by users. The evaluation of retrieval by similarity has demonstrated that the selected images extractor allowed the sorting of images according to anatomical areas. CONCLUSION: Based on these results, one can conclude that the PACS-CBIR implementation is feasible. The system has demonstrated to be DICOM-compatible, and that it can be integrated with the local information system. The similar images retrieval functionality can be enhanced by the introduction of further descriptors. Keywords: CBIR; PACS; DICOM; Medical images; Health information system.

Caritá EC, Seraphim E, Honda MO, Azevedo-Marques PM. Implementação e avaliação de um sistema de gerenciamento de imagens médicas com suporte à recuperação baseada em conteúdo. Radiol Bras. 2008;41(5):331-336.

* Trabalho realizado na Faculdade de Medicina de Ribeirão Preto da Universidade de São Paulo (FMRPUSP), Ribeirão Preto, SP, Brasil.

1. Doutor, Docente do Centro de Ciências Exatas, Naturais e Tecnológicas da Universidade de Ribeirão Preto (Unaerp), Ribeirão Preto, SP, Brasil.

2. Doutor, Docente do Instituto de Engenharia de Sistemas e Tecnologias da Informação (IESTI) da Universidade Federal de Itajubá (Unifei), Itajubá, MG, Brasil.

3. Doutor, Pós-Doutorando no Departamento de Neurologia - Subárea Biossinal e Tecnologia em Imagens - da FriedrichSchiller-Universität, Jena, Alemanha.

4. Doutor, Professor Associado do Centro de Ciências das Imagens e Física Médica do Departamento de Clínica Médica da Faculdade de Medicina de Ribeirão Preto da Universidade de São Paulo (FMRPUSP), Ribeirão Preto, SP, Brasil.

\section{INTRODUÇÃO}

Os "sistemas de comunicação e armazenamento de imagem" (picture archiving and communication systems - PACS) já se tornaram a opção tecnológica preferida para as tarefas de transmissão, armazena-

Endereço para correspondência: Dr. Edilson Carlos Caritá. Rua Arnaldo Victaliano, 1404, ap. 34, Jardim Palma Travassos. Ribeirão Preto, SP, Brasil, 14091-22. E-mail: edcarita@uol.com.br Recebido para publicação em 29/8/2007. Aceito, após revisão, em 25/2/2008. mento e visualização de dados na área de diagnóstico por imagem. Mais recentemente, devido ao grande aumento no volume de imagens gerado pelas técnicas disponíveis, o interesse na otimização dos processos de arquivamento e recuperação de informação tem-se firmado como um importante objeto de estudo na informática radiológica. Assim, enquanto o requisito clássico para um PACS seria a habilidade de visualizar imagens e informações clínicas relacionadas, um grande interesse em 
técnicas que utilizam a "recuperação de imagem baseada em conteúdo" (contentbased image retrieval - CBIR) vem surgindo nos últimos anos. Essas técnicas buscam localizar, a partir de informações extraídas do caso em estudo, casos similares anteriormente diagnosticados e armazenados. Os sistemas CBIR utilizam informações extraídas das imagens para representá-las, sendo seu principal objetivo o estabelecimento de uma descrição semântica para essas imagens ${ }^{(\mathbf{1})}$. Nesse tipo de abordagem $^{(2-5)}$, imagens são recuperadas a partir da similaridade de seus atributos, que são comparados com os atributos extraídos de uma imagem referência. Os atributos mais utilizados são cor, textura, forma, estruturas e relacionamento espacial, que servem como chaves para a recuperação das imagens dentro de uma aproximação de similaridade previamente estabelecida. A recuperação baseada em conteúdo tem-se mostrado uma alternativa importante e um complemento fundamental para os sistemas tradicionais de busca baseada em texto ${ }^{(6-8)}$. Um PACS com suporte à recuperação de imagens baseada em conteúdo (PACS-CBIR) consiste de um sistema que contempla a conexão com as modalidades de imagens, visualização, armazenamento, recuperação das imagens e laudos mediante informações alfanuméricas ou textuais, e a recuperação dos exames através de busca por similaridade das características das imagens.

Neste artigo é apresentada a implementação de uma arquitetura para o gerenciamento de imagens médicas dentro do ambiente hospitalar, que integra a função CBIR, e sua avaliação englobando as funcionalidades dos módulos voltados para o gerenciamento de imagens médicas, suporte para a recuperação baseada em conteúdo e recuperação das imagens e informações associadas por meio de dados textuais. As imagens utilizadas neste estudo foram obtidas de modalidades de imagens médicas digitais, como ressonância magnética (RM) e tomografia computadorizada (TC), em formato digital imaging and communication in medicine (DICOM), indexadas, armazenadas e vinculadas ao "sistema de informação em radiologia" (radiology information system - RIS) do hospital ${ }^{(\mathbf{9})}$, para posterior visualização em conjunto com seus respectivos laudos. As consultas às imagens e laudos podem ser realizadas por meio de palavras-chave (busca textual) ou utilizando-se de uma interface para recuperação de imagens a partir da similaridade do conteúdo pictórico, quantificada através de atributos de textura.

\section{MATERIAIS E MÉTODOS}

A modelagem da arquitetura do PACSCBIR foi criada com base nos sistemas de informação e fluxo de trabalho do hospital-escola no qual o estudo foi realizado. $\mathrm{Na}$ Figura 1 é ilustrada a modelagem da arquitetura implementada.

O servidor de imagens foi implementado utilizando-se os códigos fontes do programa PACSOne versão 3.1.8, que é um sistema de comunicação e armazenamento de imagens de código aberto, desenvolvido em 2004 por Xiaohui Li nos Estados Unidos e distribuído pela empresa RainbowFish Software ${ }^{(\mathbf{1 0})}$. O PACSOne é um servidor para armazenamento de longo e curto prazos de imagens médicas em conformidade com o padrão DICOM, que recebe as imagens das respectivas modalidades e faz seu armazenamento para posterior recuperação e distribuição. As informações das configurações do servidor e das imagens são armazenadas em um banco de dados relacional, sendo que para este estudo foi utilizado o "sistema gerenciador de banco de dados” (SGBD) MySQL versão 4.1.16, que é um software livre, portável e compatível com diversas linguagens de programação ${ }^{(11)}$.

As interfaces de recuperação textual e por similaridade de imagens foram desenvolvidas contemplando tecnologias para rede mediante linguagens hypertext markup language (HTML) e hypertext preprocessor (PHP) versão 4.0.4. Para que as imagens no formato DICOM fossem visualizadas no navegador, foi desenvolvido um aplicativo utilizando a linguagem de programação Java ${ }^{(\mathbf{1 2})}$, incluindo funções para ajuste de brilho e contraste.

O sistema CBIR foi criado para extrair as características das imagens armazenadas no servidor PACS e indexá-las para posterior recuperação por similaridade. $\mathrm{O}$ serviço responsável pela extração das características foi desenvolvido em linguagem de programação $\mathrm{C}++$, sendo executado através de linha de comando. No momento em que esse serviço é iniciado, ele verifica os exames que estão armazenados no servidor PACS, extrai as características das imagens utilizando os descritores de textura de Haralick et al. ${ }^{(\mathbf{1 3})} \mathrm{e}$ as armazena em forma de um vetor no banco de dados do módulo CBIR. A estrutura métrica utilizada como base para indexação das características extraídas é a slim-tree, proposta por Traina Jr et al. ${ }^{(\mathbf{1 4})}$.

Para a avaliação do sistema implementado observou-se seu desempenho, sua estabilidade e as principais funcionalidades

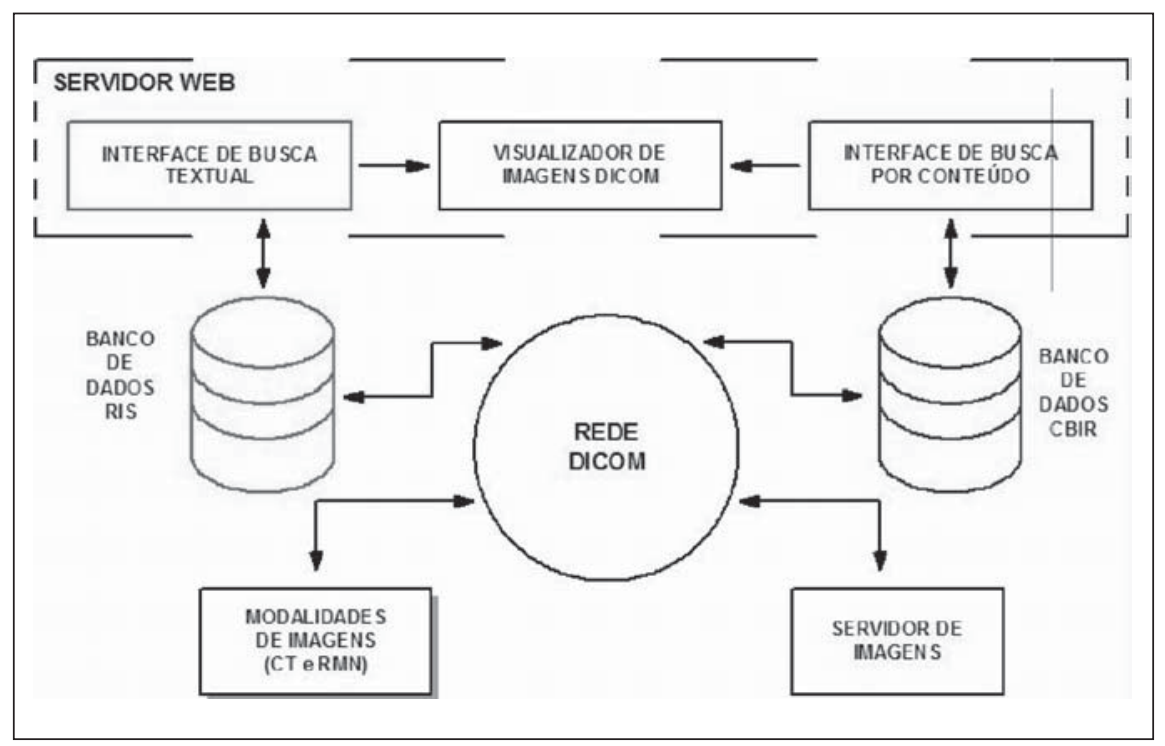

Figura 1. Arquitetura para um PACS-CBIR. 
e conformidades com o padrão DICOM. Um total de 120 exames, incluindo 60.401 imagens de RM e TC, foi utilizado para avaliação.

O sistema de recuperação textual passou por dois processos de avaliação: uma avaliação quantitativa, que teve como objetivo a validação e avaliação das funcionalidades do DICOM, para garantir que todas as imagens armazenadas no servidor PACS e vinculadas ao RIS eram disponibilizadas no momento da recuperação, juntamente com os dados demográficos do paciente e as informações do exame, e também a mensuração do tempo de retorno de cada exame; e uma avaliação qualitativa, considerando a satisfação do usuário em relação ao sistema, que foi realizada por quatro médicos e um cientista da computação. No Quadro 1 são apresentados a formação e o tempo de experiência de cada profissional. Os itens avaliados pelos usuários foram: interface do sistema, tempo de retorno dos exa- mes, tempo de retorno das imagens, qualidade das imagens, facilidade de navegação e ajuste da imagem. Os referenciais de qualidade utilizados foram: 1 (muito ruim — não adequado para uso); 2 (ruim pode ser usado com dificuldade); 3 (satisfatório - pode ser usado, porém com alguma limitação significativa); 4 (bom pode ser usado, com alguma limitação não importante); 5 (muito bom - adequado para o uso).

Para a avaliação do sistema de consulta por similaridade de imagens foram considerados somente os exames de RM, totalizando 61 casos e 17.099 imagens (39 casos e 12.939 imagens de encéfalo; 8 casos e 1.456 imagens de abdome; 10 casos e 2.448 imagens de coluna; e 4 casos e 256 imagens de faringe). Para essa avaliação, inicialmente o usuário escolhia uma modalidade de exame, definia uma imagem referência e informava o número de imagens semelhantes que o sistema deveria retornar

Quadro 1 Formação e tempo de experiência dos avaliadores do sistema.

\begin{tabular}{|clc|}
\hline Identificador & \multicolumn{1}{c|}{ Formação } & Tempo de experiência \\
\hline M1 & Médico radiologista & 15 anos \\
M2 & Médico radiologista & 13 anos \\
M3 & Médico neurorradiologista & 19 anos \\
M4 & Médico neurorradiologista & 7 anos \\
C1 & Cientista de computação & 12 anos \\
\hline
\end{tabular}

(Figura 2). Após o processo de recuperação, na avaliação do desempenho do sistema, considerou-se uma imagem recuperada como correta quando pertencente à mesma região anatômica da imagem de referência ou quando possuindo a mesma condição diagnóstica (normal ou não). Para exames com laudo não-normal verificouse a compatibilidade das descrições diagnósticas presentes nos laudos.

\section{RESULTADOS}

O sistema para recuperação textual foi implementado para permitir a integração entre o servidor de imagens, o servidor do RIS e o visualizador de imagens, admitindo que o usuário autorizado consulte as informações dos pacientes, seus exames e suas respectivas imagens. No Quadro 2 são apresentadas as opções de consulta textual disponíveis no sistema. Na Figura 3 é apresentada a tela para realizar uma consulta complexa. A partir das informações do paciente e da lista dos exames realizados, é possível acessar e visualizar as imagens (Figura 4).

Os resultados da avaliação qualitativa do sistema de recuperação textual são apresentados na Tabela 1 . Na Tabela 2 são apresentados os resultados obtidos nas consultas realizadas para avaliar a similaridade

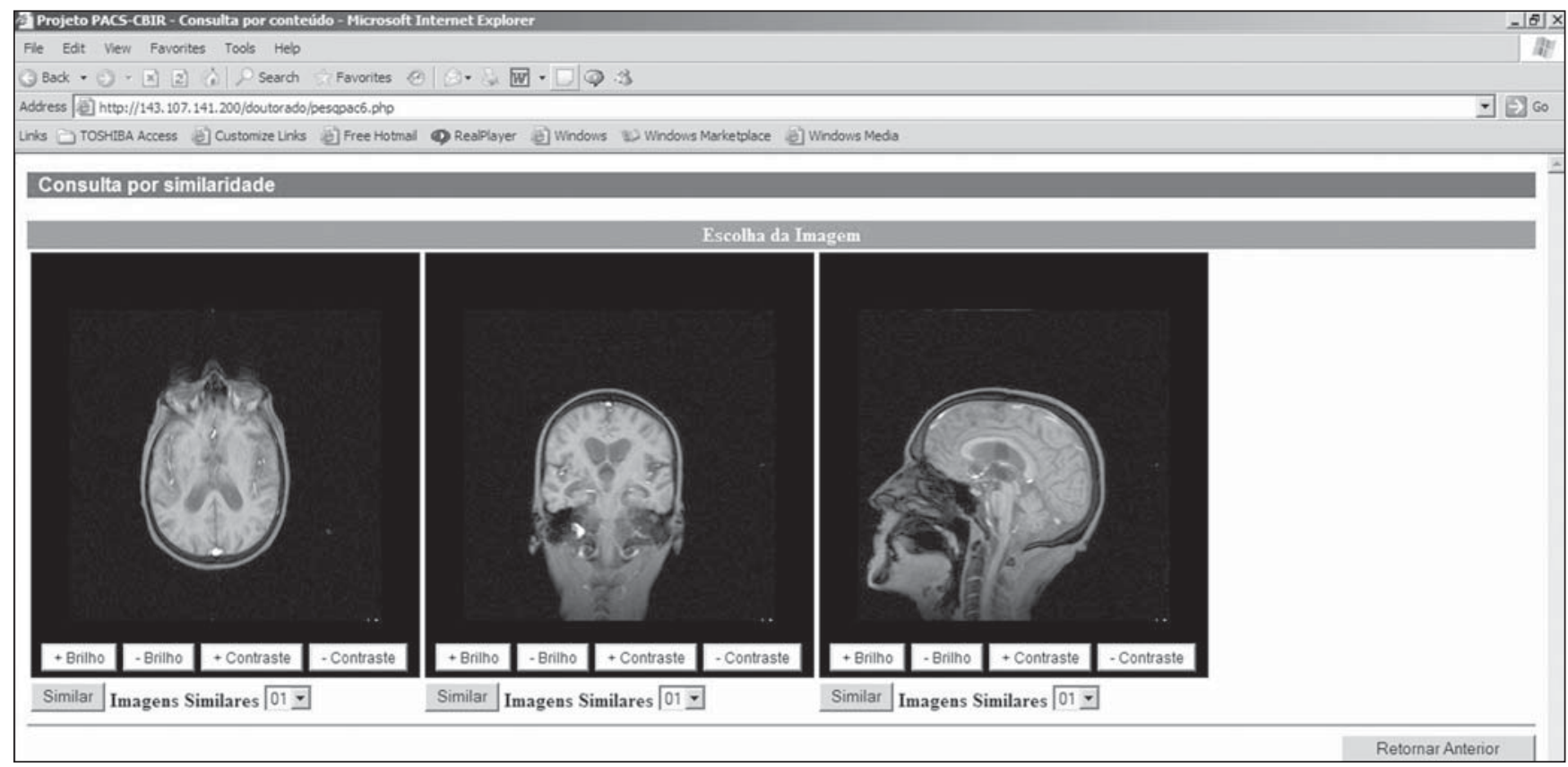

Figura 2. Interface para escolha da imagem de referência para consulta por similaridade. 


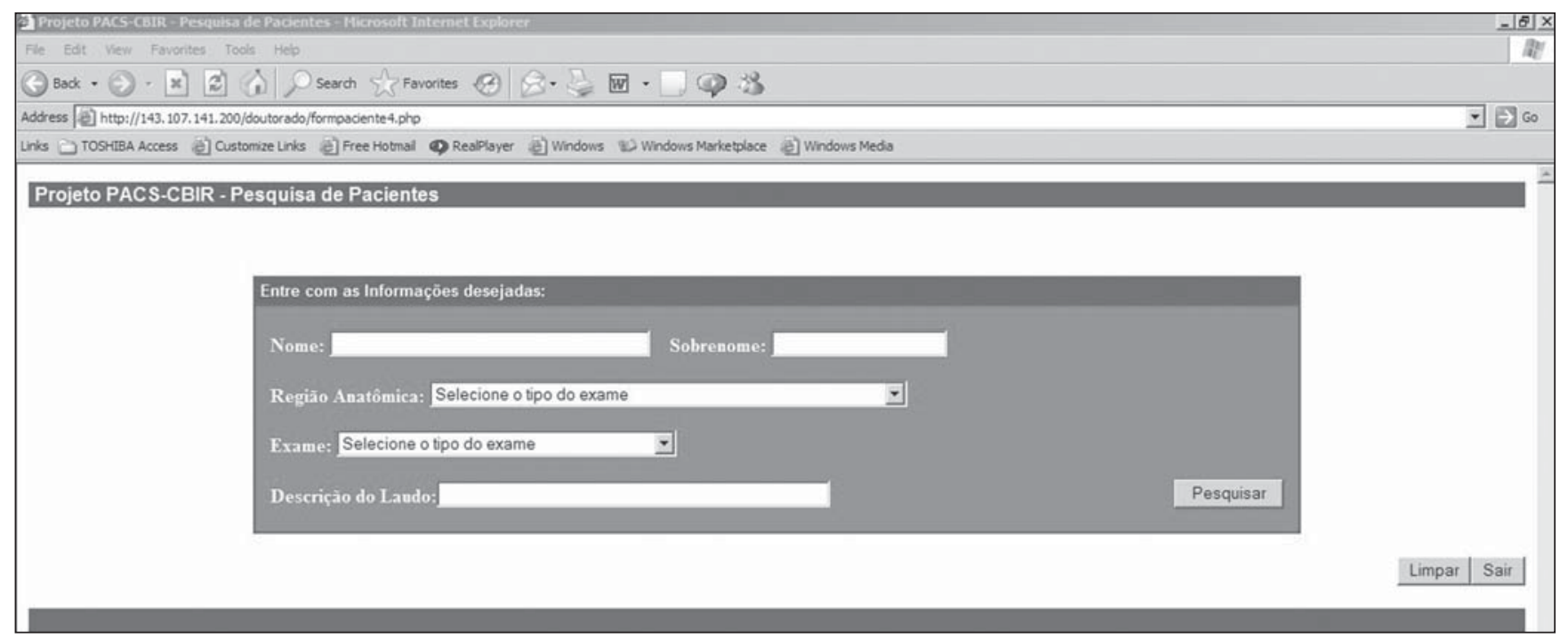

Figura 3. Tela da opção consulta complexa do módulo de consulta textual.

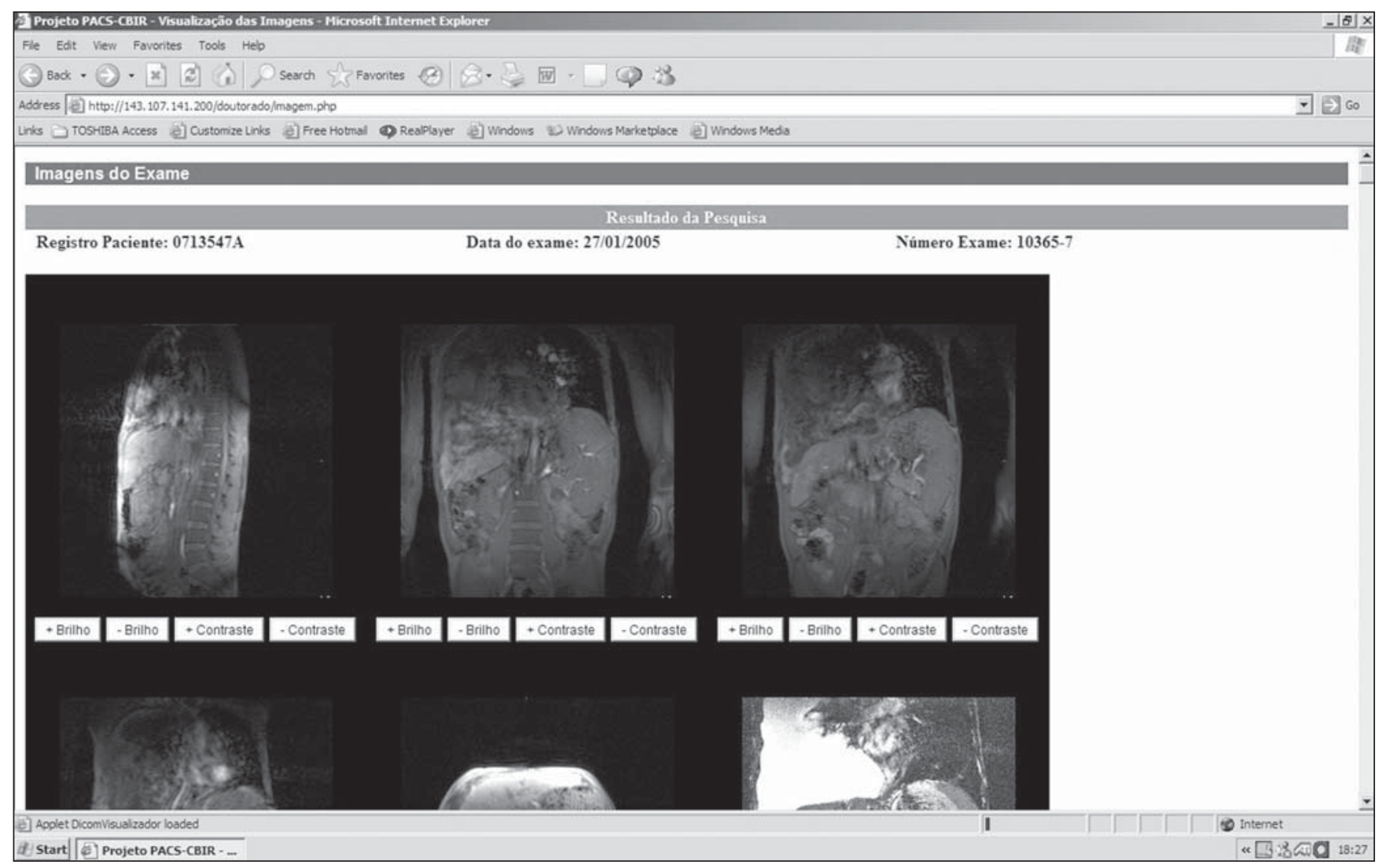

Figura 4. Imagens de um exame apresentadas pelo visualizador DICOM implementado.

das imagens recuperadas através dos descritores de textura utilizados, considerando a região anatômica. Na coluna "Média" é apresentada a porcentagem média de imagens corretas que o sistema retornou para cada região anatômica. Observou-se valor médio geral de acertos de $72 \%$ e desviopadrão de 0,84 .

Na Tabela 3 são apresentados os resultados validando a conclusão do laudo (normal ou não) e a compatibilidade das descrições dos laudos para os casos não-nor- mais para cada região anatômica. Observou-se valor médio geral de acertos de $81 \%$ e desvio-padrão de 1,47 para a conclusão do laudo, e valor médio geral de acertos de $64 \%$ e desvio-padrão de 1,13 para a compatibilidade da descrição do laudo. 
Quadro 2 Opções de consulta textual do sistema.

\begin{tabular}{|ll|}
\hline \multicolumn{1}{|c|}{ Opções } & \multicolumn{1}{c|}{ Descrição } \\
\hline Nome/sobrenome & $\begin{array}{l}\text { O usuário deverá informar o nome e/ou sobrenome do paciente, para que o sistema possa encontrar o paciente com os } \\
\text { dados informados e, posteriormente, recuperar seus exames e respectivas imagens }\end{array}$ \\
Região anatômica/exame & $\begin{array}{l}\text { O usuário informará a região anatômica e/ou o tipo de exame e um período e o sistema recuperará os exames e as respec- } \\
\text { tivas imagens } \\
\text { O usuário informará o identificador do paciente e o sistema recuperará as informações do paciente, bem como seus exa- } \\
\text { mes e as respectivas imagens, mediante uma busca única } \\
\text { O usuário deverá informar palavras-chave da conclusão do laudo e um período e o sistema recuperará os exames e as res- } \\
\text { pectivas imagens } \\
\text { Através dessa opção o usuário poderá fazer uma mescla entre os atributos nome, sobrenome, região anatômica, tipo do } \\
\text { Consulta }\end{array}$ \\
\hline
\end{tabular}

Tabela 1 Resultados da avaliação qualitativa do sistema de recuperação textual.

\begin{tabular}{lccccccc}
\hline \multicolumn{1}{c}{ Item } & M1 & M2 & M3 & M4 & C1 & Média & Conclusão final \\
\hline Interface do sistema & 4 & 5 & 5 & 5 & 5 & 4,8 & Muito bom \\
Tempo de retorno dos exames & 3 & 5 & 5 & 5 & 5 & 4,6 & Muito bom \\
Tempo de retorno das imagens & 3 & 5 & 4 & 5 & 4 & 4,2 & Bom \\
Qualidade da visualização das imagens & 4 & 4 & 4 & 4 & 4 & 4 & Bom \\
Facilidade de navegação & 4 & 5 & 5 & 5 & 5 & 4,8 & Muito bom \\
Ajuste da imagem & 3 & 3 & 3 & 3 & 3 & 3 & Satisfatório \\
\hline
\end{tabular}

M1 e M2, médicos radiologistas; M3 e M4, médicos neurorradiologistas; C1, cientista de computação.

Tabela 2 Resultados obtidos considerando a região anatômica retornada.

\begin{tabular}{ccc}
\hline Região anatômica & Média & Desvio-padrão \\
\hline Encéfalo & $80 \%$ & 1,41 \\
Abdome & $100 \%$ & 0 \\
Coluna & $20 \%$ & 0 \\
Faringe & $80 \%$ & 1,41 \\
\hline
\end{tabular}

Tabela 3 Resultados obtidos considerando a conclusão e a compatibilidade da descrição do laudo.

\begin{tabular}{ccccc}
\hline Região anatômica & Média (laudo) & Desvio-padrão & Média (compatibilidade) & Desvio-padrão \\
\hline Encéfalo & $72 \%$ & 1,14 & $56 \%$ & 0,70 \\
Abdome & $80 \%$ & 0 & $50 \%$ & 0 \\
Coluna & $100 \%$ & 0 & $100 \%$ & 0 \\
Faringe & $70 \%$ & 2,12 & $50 \%$ & 2,12 \\
\hline
\end{tabular}

\section{DISCUSSÃO}

Considerando-se a avaliação qualitativa do sistema de recuperação textual, observa-se que no item "interface do sistema" quatro profissionais atribuíram nota 5 e um profissional, nota 4; assim, este item pode ser considerado muito bom. Na implementação das interfaces do projeto PACSCBIR (interface do PACS e interfaces de recuperação textual e baseada em conteúdo) seguiu-se a estratégia mundial para disponibilizar informações de maneira segura, prática e rápida, mediante desenvolvimento de sistemas de informação que utilizam tecnologias para Internet. Assim, o usuário não precisa ter o sistema instalado em sua máquina e pode acessá-lo de qualquer lugar. No item "tempo de retorno dos exames", quatro profissionais atribuíram nota 5 e um profissional, nota 3; a maioria dos profissionais considerou o tempo de retorno dos exames muito bom. Ressalta-se que esse tempo não ultrapassou cinco segundos, todavia, variações de velocidade na taxa de transferência podem ocorrer em função do tráfego da rede de computadores do hospital.
$\mathrm{Na}$ avaliação do item "tempo de retorno das imagens", dois profissionais atribuíram nota 5 , dois profissionais atribuíram nota 4 e um profissional, nota 3 , sendo possível considerar este item como bom; entretanto, o tempo médio de retorno das imagens ficou sempre no intervalo de 10 a 15 segundos. Embora esse aspecto não tenha sido quantificado neste trabalho, é provável que o maior tempo de espera esteja associado ao processo de montagem da tela para exibição das imagens e não com o processo de consulta pelo módulo CBIR. Isso estaria em conformidade com os resultados apresentados por Harrison ${ }^{(15)}$, responsável pela implantação de um PACS-Web no Centro Médico da Universidade de Mississipi, em Jackson, nos Estados Unidos. Segundo o autor, a consulta é rápida e o que é computacionalmente dispendioso é retornar as imagens da base de dados e montar a tela de visualização.

No item "qualidade de visualização das imagens", todos os profissionais atribuíram nota 4 , conceito bom, sendo a principal limitação encontrada relativa ao ajuste do janelamento para exibição das imagens. Essa limitação é confirmada na avaliação do item "ajuste da imagem", para o qual todos os profissionais atribuíram nota 3, sendo sugerido o acréscimo de algumas ferramentas como zoom, segmentação e binarização.

Considerando-se a análise do item "facilidade de navegação", quatro profissionais atribuíram nota 5 e um profissional, nota 4. A maioria dos profissionais considerou este item muito bom, obtendo-se um resultado compatível com outros trabalhos relativos à utilização de tecnologias para Internet em PACS. Citando Harrison ${ }^{(15)}$, 
Peer et al. ${ }^{(16)}$ e Cao et al. ${ }^{(17)}$, as principais vantagens do uso dessas tecnologias são a redução de custos e a descentralização dos exames, permitindo fácil acesso dos radiologistas às imagens pela rede do hospital ou pela Internet.

Os resultados obtidos com a avaliação do desempenho do sistema de recuperação de imagens similares utilizando atributos de textura, com valor médio geral de acerto de $72 \%$, são compatíveis com resultados encontrados em outros trabalhos publica$\operatorname{dos}^{(\mathbf{1}, \mathbf{1 8 , 1 9 )}}$. Pereira Jr et al. ${ }^{(\mathbf{1 8})}$ apresentam um estudo mostrando que atributos de textura podem ser úteis para separar automaticamente regiões normais de regiões contendo nódulos ou microcalcificação em mamografias digitalizadas, com acertos acima de $90 \%$. Porém, são muito pouco eficientes para distinguir lesões malignas de benignas, com seu nível de acerto, nesse caso, caindo para a ordem de $50 \%$. Em um trabalho envolvendo análise de textura para recuperação de imagens por similaridade, Oliveira et al. ${ }^{(19)}$ relatam resultados de precisão na ordem de $54 \%$ para imagens sagitais de joelho e $40 \%$ para imagens axiais de cabeça. Kinoshita et al. ${ }^{(\mathbf{1})}$ apresentam resultados da ordem de $78 \%$ a $83 \%$ de acerto em um sistema CBIR, baseado em textura e redes neurais artificiais, desenvolvido para recuperar imagens mamográficas por similaridade de densidade de tecido.

\section{CONCLUSÕES}

A arquitetura implementada foi desenvolvida tendo-se por base tecnologias não proprietárias de código livre e aberto, as características do sistema de informação e do fluxo de exames do hospital onde o projeto foi implementado. Porém, sua estrutura pode ser adaptada para funcionar em conjunto com qualquer sistema de gerenciamento e modalidade de geração de imagens médicas que estejam em conformidade com o padrão DICOM, basicamente adicionando-se o módulo de recuperação de imagens por similaridade de conteúdo. Para implementação do algoritmo de recu- peração por similaridade foram utilizados descritores de textura, que fornecem medidas de propriedades de regiões como suavidade, rugosidade e regularidade ${ }^{(20)}$. Porém, o desempenho do sistema pode ser melhorado com a inclusão de outros descritores, voltados para caracterização de forma, por exemplo.

Do ponto de vista de sua aplicação, o sistema implementado pode ser utilizado na rotina clínica como ferramenta de auxílio à tomada de decisão, conforme descrito no trabalho publicado por Huang et al. ${ }^{(21)}$, que descreve a implementação e avaliação de uma ferramenta de suporte ao diagnóstico com base na recuperação de imagens similares junto ao Childrens Hospital Los Angeles. O estudo envolveu 2.500 exames consecutivos de RM de encéfalo de crianças, com resultados variando de $10 \%$ a $60 \%$ de acerto nas consultas por similaridade, sendo que a doença melhor caracterizada foi a neurofibromatose $(60 \%)$ e a pior caracterizada, o cisto aracnóide (10\%). É evidente que, em relação ao fluxo de trabalho, a utilização de um sistema CBIR como ferramenta de auxílio ao diagnóstico irá impactar negativamente na produtividade do serviço, porém, seus benefícios ficam bastante evidentes como ferramenta de apoio à pesquisa e ao ensino em radiologia, conforme descrito no artigo de revisão publicado por Müller et al. ${ }^{(\mathbf{8})}$. Ressalta-se, finalmente, que na literatura ainda há poucos relatos voltados para a integração de ferramentas de recuperação por conteúdo em sistemas de gerenciamento de imagens, reforçando o caráter inovador do trabalho descrito neste artigo.

\section{REFERÊNCIAS}

1. Kinoshita SK, Azevedo-Marques PM, Pereira RR $\mathrm{Jr}$, et al. Content-based retrieval of mammograms using visual features related to breast density patterns. J Digit Imaging. 2007;20:172-90.

2. Song BC, Kim MJ, Ra JB. A fast multiresolution feature matching algorithm for exhaustive search in large image databases. IEEE Trans Circuits Syst Video Technol. 2001;11:673-8.

3. Cai W, Feng DD, Fulton R. Content-based retrieval of dynamic PET functional images. IEEE Trans Inf Technol Biomed. 2000;4:152-8.
4. Assfalg J, Pala P. Querying by photographs: a VR metaphor for image retrieval. IEEE Multimedia. 2000;7:52-9.

5. Wang Z, Chi Z, Feng D. Content-based image retrieval using block-constrained fractal coding and nona-tree decomposition. IEEE Proc Vis Image Signal Process. 2000;147:9-15.

6. Alto H, Rangayyan RM, Desautels JEL. Contentbased retrieval and analysis of mammographic masses. J Electron Imaging. 2005;14:1-17.

7. Vailaya A, Figueiredo MAT, Jain AK, et al. Image classification for content-based indexing IEEE Trans Image Processing. 2001;10:117-30.

8. Müller H, Michoux N, Bandon D, et al. A review of content-based image retrieval systems in medical applications - clinical benefits and future directions. Int J Med Inform. 2004;73:1-23.

9. Azevedo-Marques PM, Caritá EC, Benedicto AA, et al. Integração RIS/PACS no Hospital das Clínicas de Ribeirão Preto: uma solução baseada em "web". Radiol Bras. 2005;38:37-43.

10. PacsOne. [Acessado em: 10/6/2007]. Disponível em: http://www.pacsone.net

11. Milani A. MySQL - guia do programador. São Paulo: Novatec; 2006.

12. Caritá EC, Matos ALM, Azevedo-Marques PM Ferramentas para visualização de imagens médicas em hospital universitário. Radiol Bras. 2004;37:437-40

13. Haralick RM, Shanmugan K, Dinstein I. Textural features of images classification. IEEE Trans Systems Man Cybernetics. 1973;3:610-21.

14. Traina C Jr, Traina AJM, Faloutsos C, et al. Fast indexing and visualization of metric data sets using slim-trees. IEEE Trans Knowledge Data Engineering. 2002;14:244-60.

15. Harrison SW. Success with Web-based image access. Radiol Manage. 2003;25:36-8.

16. Peer S, Vogl R, Peer R, et al. Sophisticated hospital information system/radiology information system/picture archiving and communications system (PACS) integration in a large-scale traumatology PACS. J Digit Imaging. 1999;12:99102.

17. Cao F, Huang HK, Zhou XQ. Medical image security in a HIPAA mandated PACS environment Comput Med Imaging Graph. 2003;27:185-96.

18. Pereira RR Jr, Azevedo-Marques PM, Honda MO, et al. Usefulness of texture analysis for computerized classification of breast lesions on mammograms. J Digit Imaging. 2007;20:248-55.

19. Oliveira MC, Azevedo-Marques PM, Cirne Filho WC. Grades computacionais na otimização da recuperação de imagens médicas baseada em conteúdo. Radiol Bras. 2007;40:255-61.

20. Gonzalez RC, Woods RE. Processamento de imagens digitais. São Paulo: Edgard Blücher; 2000.

21. Huang HK, Nielsen JF, Nelson MD, et al. Imagematching as a medical diagnostic support tool (DST) for brain diseases in children. Comput Med Imaging Graph. 2005;29:195-202. 